\title{
New Approach for Image Fusion Based on Curvelet Approach
}

\author{
Gehad Mohamed Taher \\ Computer Science \\ Faculty of Computer and Informatics \\ Suez Canal University \\ Ismailia, Egypt
}

\author{
Mohamed ElSayed Wahed \\ Computer Science \\ Faculty of Computer and Informatics \\ Suez Canal University \\ Ismailia, Egypt
}

\author{
Ghada EL Taweal \\ Computer Science \\ Faculty of Computer and Informatics \\ Suez Canal University \\ Ismailia, Egypt
}

\begin{abstract}
Most of the image fusion work has been limited to monochrome images. Algorithms which utilize human colour perception are attracting the image fusion community with great interest. It is mainly due to the reason that the use of colour greatly expands the amount of information to be conveyed in an image. Since, the human visual system is very much sensitive to colours; research was undertaken in mapping three individual monochrome multispectral images to the respective channels of an RGB image to produce a false colour fused image. Producing a fused colour output image which maintains the original chromaticity of the input visual image is highly tricky.
\end{abstract}

The focus of this paper is developing a new approach to fuse a color image (visual image) and a corresponding grayscale one (Infrared image - IR) using the curvelet approach using different fusion rules in new fields. The fused image obtained by the proposed approach maintain the high resolution of the colored image (visual image), incorporate any hidden object given by the IR sensor as an example, or complements the two input images and keep the natural color of the visual image.

Keywords-Image fusion; visual colored image; monochrome images

\section{INTRODUCTION}

Image fusion is a process of combining complementary information from multiple sensor images to generate a single image that contains a more accurate description of the scene than any of the individual images. As for example while MMW (millimeter wave) sensors have many advantages, the low cost IR makes the study of fusing visual and IR images of great interest.

In our work, we are using fusion to help human or computer to detect the hidden objects using IR and visual sensors. Most of the image fusion work has been limited to monochrome images [1]. However, based on biological research results, the human visual system is very sensitive to colors. Waxman, Aguilar [4-6]. Al use a neural network to fuse a low- light visible image and IR image to generate a three channel false color image used for night operations. In addition, Aguilar [7] has extended their work to fuse multimodality volumetric imagery.

In this paper, we introduce a new approach to fuse a color visual image with a corresponding grayscale IR image or any other sensor; using the proposed approach the fused image will maintain the high resolution and the natural color of the visual image.

The paper is organized as follows, section 2 describes the proposed image fusion approach, and section 3 presents the experimental results which demonstrate the feasibility of the proposed fusion approach, section 4 conclusion.

\section{CURVELET TRANSFORM}

The curvelet transform is a very young signal analysing method with good potential. It is recognized as a milestone on image processing and other applications [2].

So why we use curvelet; actually, the time frequency analysis is decomposed a signal to several orthogonal bases. We can quantize the signal to the summation of different basis with different coefficient:

$$
\mathrm{f}=\sum_{\mathrm{k}} \mathrm{a}_{\mathrm{k}} \mathrm{b}_{\mathrm{k}}
$$

Curvelet Coefficients basis. frame

Curvelet transform is more accurate to deal with the curve than wavelet transform the below Fig. 1 shows this.

\section{Wavelet approach}

Many wavelet coefficients are needed to account edges.

i.e. singularities along lines or curves needed to account edges.

\section{Curvelet approach}

Less coefficients are needed to account edges.
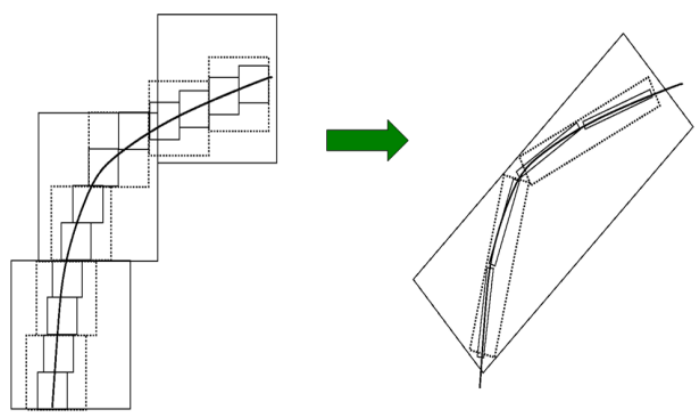

Fig. 1. Difference between curvelet and wavelet approach 
The overview of the curvelet transform is shown below for four step:

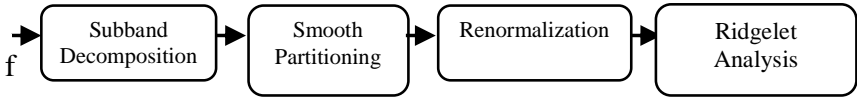

\section{A. Subband Decomposition}

We define a bank of subband filter $\mathrm{P} 0,(\triangle \mathrm{s}, \mathrm{s} \geqq 0)$. The object $\mathrm{f}$ is filter into subbands:

$$
f \mapsto\left(P_{0} f, \Delta_{1} f, \Delta_{2} f, \ldots\right)
$$

This step divides the image into several resolution layers. Each layer contains details of different frequencies:

P0 $\rightarrow$ Lowpass filter

$\Delta 1, \Delta 2, \rightarrow$ Band-pass (high-pass)

filters

\section{B. Smooth Partitioning}

Let $w$ be a smooth windowing function so by applying it to the decomposition we get

$$
h_{Q}=w_{Q} \cdot \Delta_{s} f
$$

\section{Renormalization}

In this stage of the procedure, each 'square' resulting in the previous stage is renormalized to unit scale:

$$
g_{Q}=T_{Q}^{-1} h_{Q}
$$

\section{Ridgelet construction}

The ridgelet consturction divides the frequency domain to dyadic coronae Fig.2. In the angular direction, it samples the sth corona at least $2 \mathrm{~s}$ times. In the radial direction, it samples using local wavelets. [3]

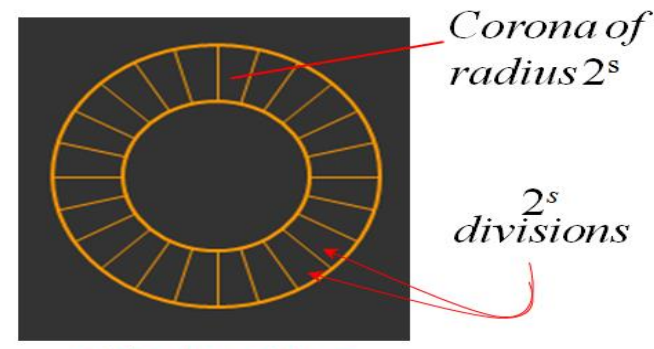

Ridgelet Tiling

Fig. 2. Ridgelet Tiling and Fourier
Transform of the Curvelet Transform each normalized square is analyzed in the ridgelet system:

$$
\alpha_{(Q, \lambda)}=\left\langle g_{Q}, p_{\lambda}\right\rangle
$$

\section{FUSION APPROACH FOR GRAY AND VISUAL IMAGES}

The proposed image fusion approach is illustrated in Fig. 3.

This method is based on the color space transform RGB HSI - RGB; The HSI is based on the RGB true color space. An RGB color image is given by an $M \times N \times 3$ array of color pixels, where each pixel is a triplet corresponding to the red, green and blue component of an RGB image at a specified location.

In HSI color space, the hue component represents the dominant color present in an image. The saturation component indicates the amount of purity. The intensity component gives the gray level values of the image.

The HSI color system is considerably closer than RGB system to human perception in describing the color sensations. Further, HSI color space allows the de-coupling of intensity component from the color carrying information in an image. Hence HSI color space is used for intermediate processing in an image fusion task.

\section{A. Steps of the proposed approach:}

1) Input two images of the same scene one grayscale and one color image.

2) The RGB components of color image (Image 1) are converted to HSI components where

$$
\mathrm{I}=(\mathrm{R}+\mathrm{G}+\mathrm{B}) / 3
$$

$\mathrm{H}=(\mathrm{B}-\mathrm{R}) / 3(\mathrm{I}-\mathrm{R}) \quad, \mathrm{S}=(1-\mathrm{R}) / \mathrm{I} \quad$ when $\mathrm{R}=$ Minimum

$(\mathrm{R}, \mathrm{G}, \mathrm{B})$

$\mathrm{H}=(\mathrm{R}-\mathrm{G}) / 3(\mathrm{I}-\mathrm{G}) \quad, \mathrm{S}=(1-\mathrm{G}) / \mathrm{I} \quad$ when $\mathrm{G}=$ Minimum

$(\mathrm{R}, \mathrm{G}, \mathrm{B})$

$\mathrm{H}=(\mathrm{G}-\mathrm{B}) / 3(\mathrm{I}-\mathrm{B}) \quad, \mathrm{S}=(1-\mathrm{B}) / \mathrm{I} \quad$ when $\mathrm{B}=$ Minimum (R, G, B)

3) Curvelet transform is applied to intensity of image 1 and the other grayscale image 2 (IR image) respectively using the Wrapping Algorithm.

4) Three different fusion rules are applied to the coefficients that are PCA, wavelet, and mean fusion rules at each location of the input images to produce a single set of coefficients in the fused output.

5) Inverse curvelet transform is applied to get the final fused output.

6) Now hue, saturation components are added to the intensity image to get the final fused color image.

7) Finally, the HSI color space is converted to RGB format. 


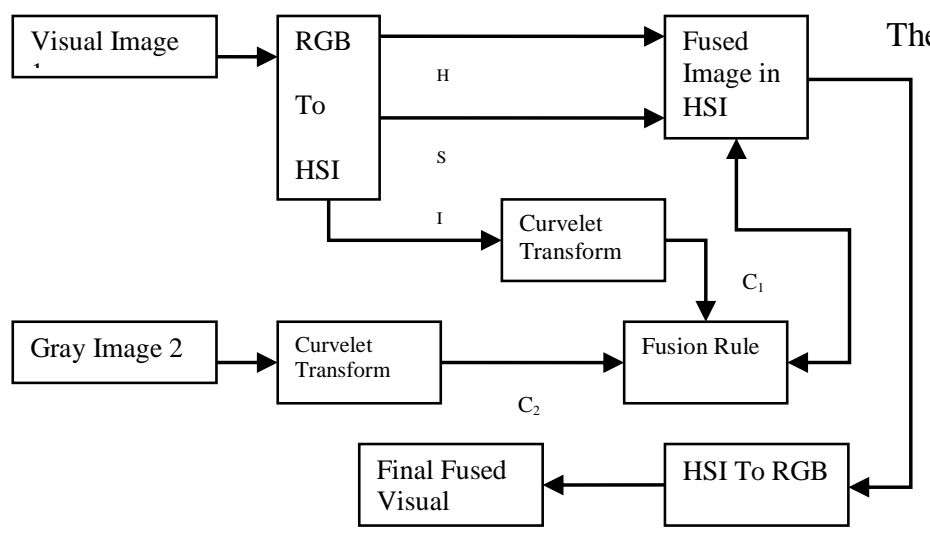

The below Fig. 4 shows this results.

Example 1:

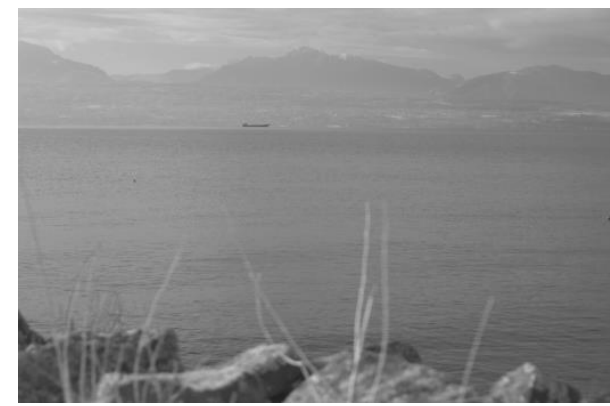

Fig. 3. Block diagram for the proposed approach

input image1 monochrome

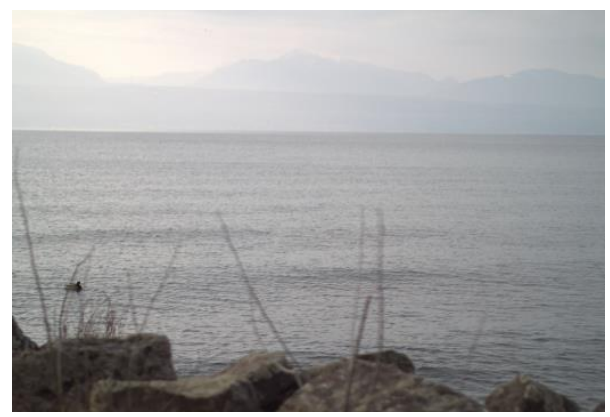

Input image 2 visual

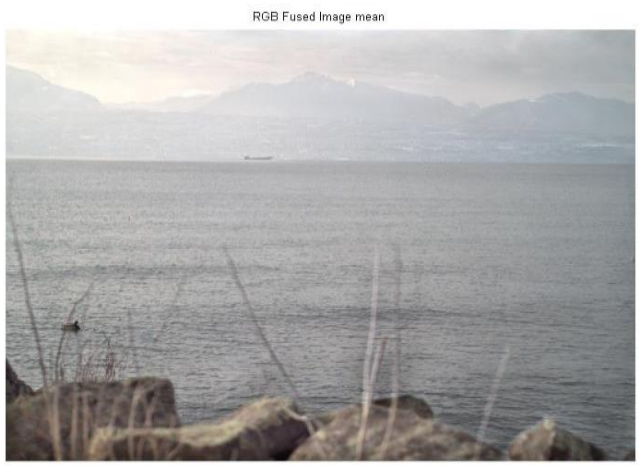

In order to illustrate the efficiency of the proposed image fusion approach, a dozen images were used in the experimental tests.

\section{A. Data Set}

By applying the experimental test for a dataset for more than 10 pair of images one is visual and the other is gray scale with the same size, as the resolution of the visual image is much higher than that of the gray scale, but the visual one doesn't convoy all of the information in the gray one. So by fusing both we got a new one that is high resolution from the visual and all of the things appear from the gray one.

\section{B. Qualitative performance comparison}

By applying the test to a pair of 4 images we found that first the visual appearance of the resulted fusion image is much better than the two input image for the three different fusion rules mean, PCA, wavelet.

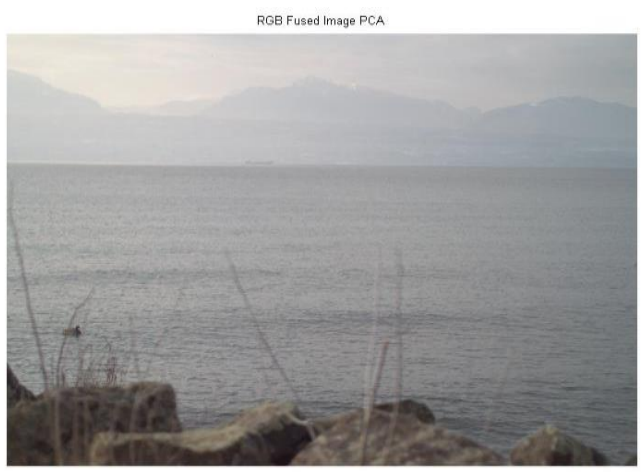



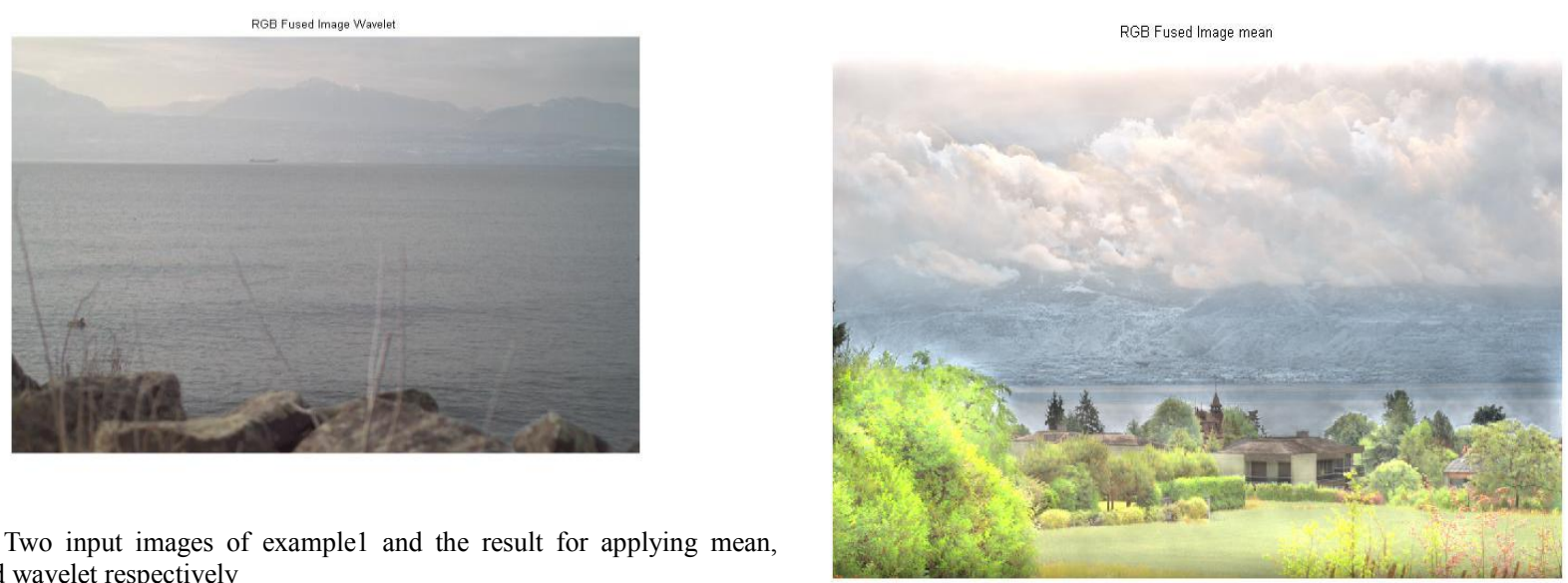

Fig. 4. Two input images of example1 and the result for applying mean, PCA and wavelet respectively

In this example the mean fusion rule is more accurate and have better resolution than the two other fusion rule.

\section{Example 2:}

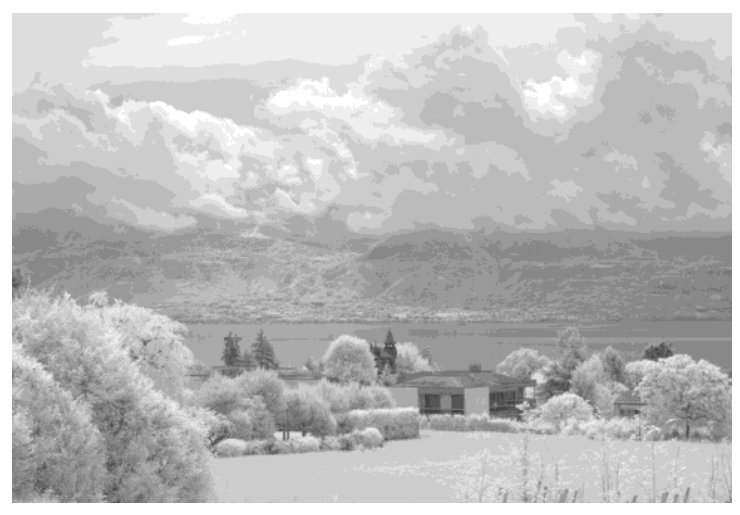

input image1 monochrome

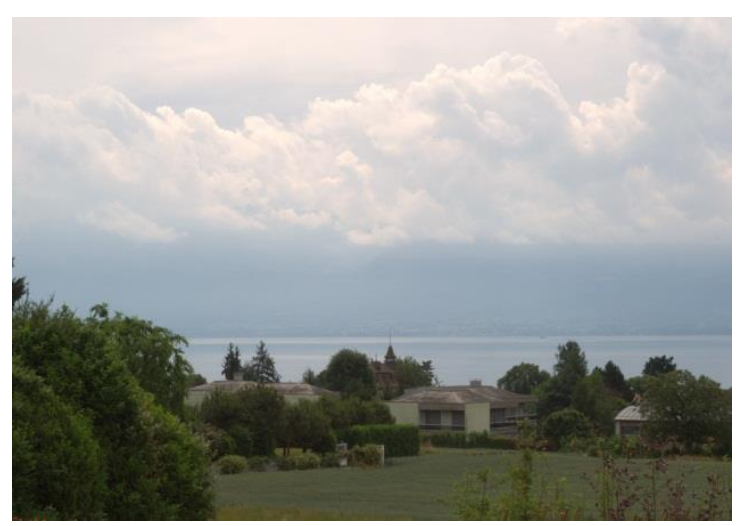

Input image2 visual
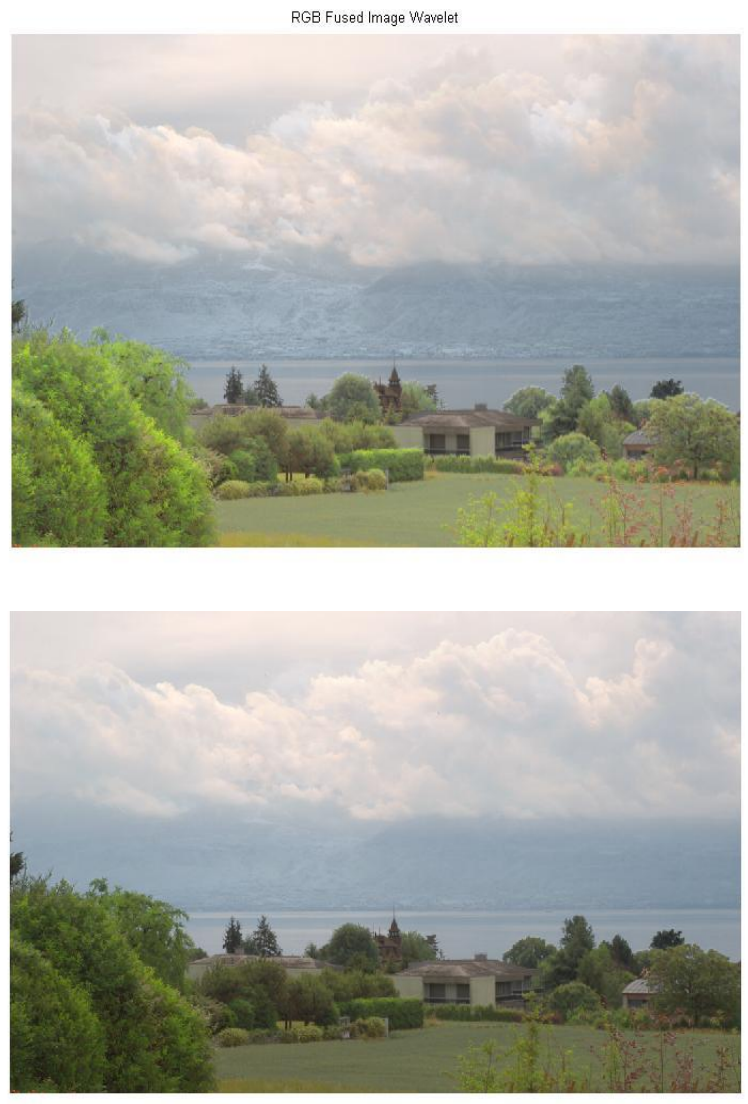

Fig. 5. Two input images of example2 and the result for applying mean, PCA and wavelet respectively

As a result for this fusion the mean fusion rule get all the details from the greyscale image but light the colours of the visual image, the other two fusion rule didn't get all the fine details from the greyscale one. 


\section{Quantitative performance comparison}

Quantitative performance is the one which involve predefined quality indicators for measuring the spectral and the spatial similarities between the fused image and the original image. [3] Here we use quality measures like mean square error, peak signal to noise ratio, entropy and standard deviation. [9]

\section{a) Root Mean square error}

The RMSE between a reference image $\mathrm{R}$ and the fused image $\mathrm{F}$ is given by There are different approaches to construct reference image using input images. In our experiments, we used the following procedure to compute RMSE. First, RMSE value $\mathrm{El}$ is computed between source image $\mathrm{A}$ and fused image F.

$$
E 1=\sqrt{\frac{1}{M N} \sum_{i=1}^{M} \sum_{j=1}^{N}(R(i, j)-F(i, j))^{2}}
$$

Smaller RMSE value indicates good fusion quality.

\section{b) Peak signal to noise ration}

The ratio between maximum possible power of the signal to the power of the corrupting noise that creates distortion of image. The peak signal to noise ratio can be represented as

$$
P S N R=10 \times \log _{10}\left(\frac{(255 \times M \times N)}{\sum \sum(x(i, j)-y(i, j))^{2}}\right.
$$

Where $\mathrm{x}$ - fused image, $\mathrm{y}$ - perfect image, $\mathrm{i}$ - pixel row index, $\mathrm{j}$ - pixel column index, $\mathrm{M}, \mathrm{N}-$ Number of rows and columns respectively.

\section{Entropy:}

The entropy of an image is a measure of information content .The estimate assumes a statistically independent source characterized by the relative frequency of occurrence of the elements in X, which is its histogram. For a better fused image, the entropy should have a larger value. A high value of entropy denotes more information content and vice versa. [8]

$$
H(S)=-\sum P(X) \log P(X)
$$

\section{Standard deviation:}

The standard deviation (SD) provides a way to determine regions which are clear and vague, it is the square root of variance, reflects the spread in the data. Thus, a high contrast image will have a larger variance, and a low contrast image will have a low variance. [8]

$$
s=\sqrt{\frac{1}{n} \sum_{i=1}^{n}\left(x_{i}-\bar{x}\right)^{2}}
$$

Where

$$
\bar{x}=\frac{1}{n} \sum_{i=1}^{n} x_{i}
$$
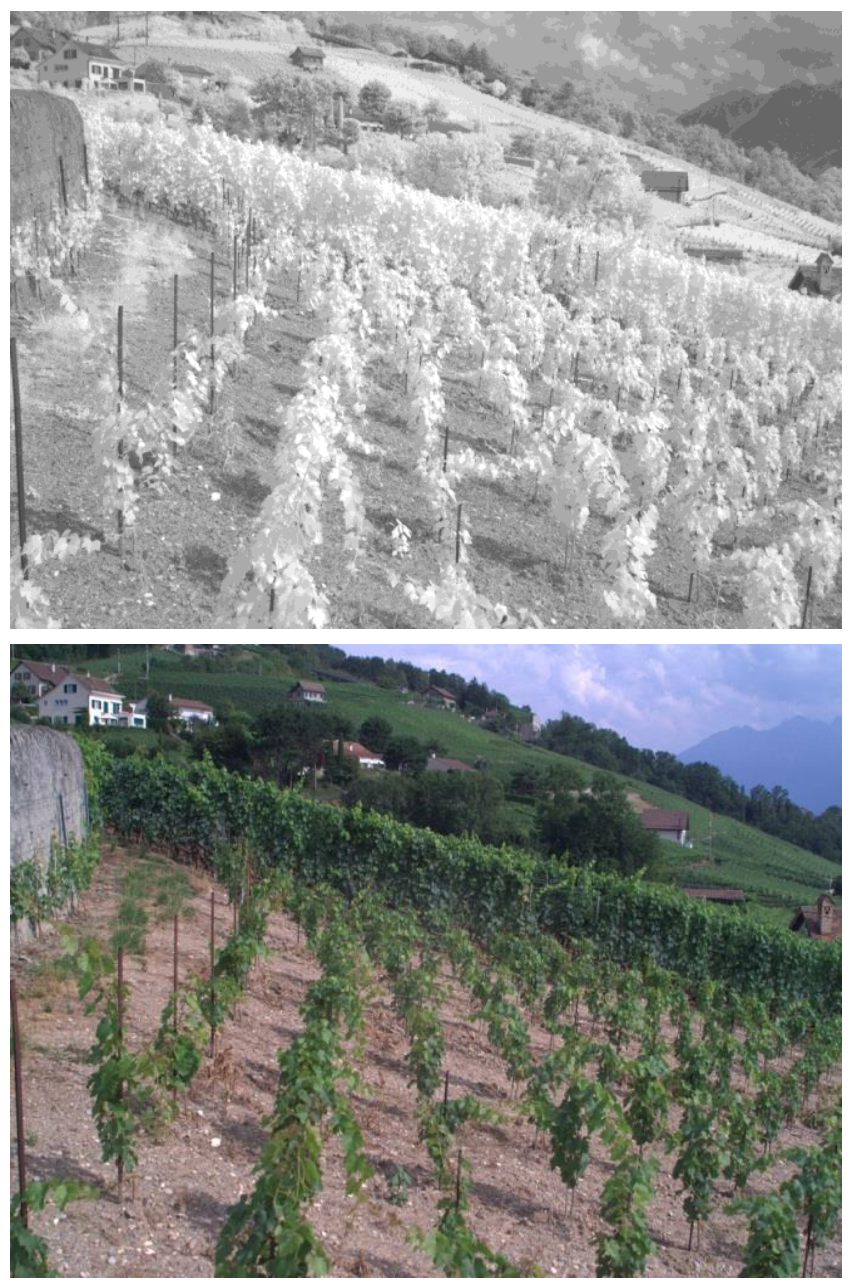

Fig. 6. Two input images one greyscale and the other visual image of the country scene

Applying these metrics to the first pair of images in Fig. 6 we get those results in table 1

TABLE I. RESULTS OF APPLYING RMSA, PSNR, ENTROPY AND SD TO IMAGES OF FIG. 6

\begin{tabular}{||c|c|c|c|c||}
\hline & RMSA & PSNR & Entropy & SD \\
\hline $\begin{array}{c}\text { PCA } \\
\text { fusion } \\
\text { rule }\end{array}$ & $1.4237 \mathrm{e}+004$ & 6.5966 & 7.6036 & 0.2384 \\
\hline $\begin{array}{c}\text { Mean } \\
\text { fusion } \\
\text { rule }\end{array}$ & 664.5194 & 19.9057 & 7.2583 & 0.1685 \\
\hline $\begin{array}{c}\text { Wavelet } \\
\text { fusion } \\
\text { rule }\end{array}$ & $2.1859 \mathrm{e}+003$ & 14.7346 & 7.0864 & $\mathbf{0 . 1 3 0 4}$ \\
\hline
\end{tabular}




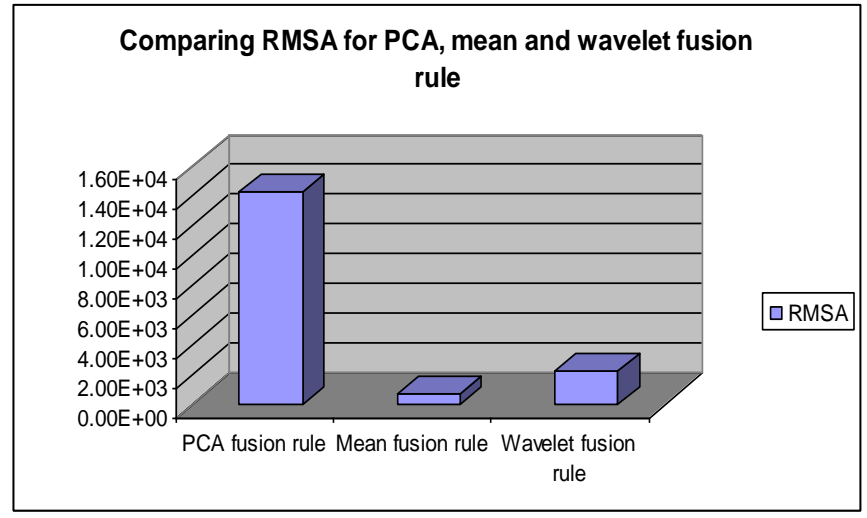

Fig. 7. RMSA for PCA, mean and wavelet fusion rule for table 1, mean fusion gives the low RMSA indicating the best fusion quality than the PCA and wavelet fusion rule

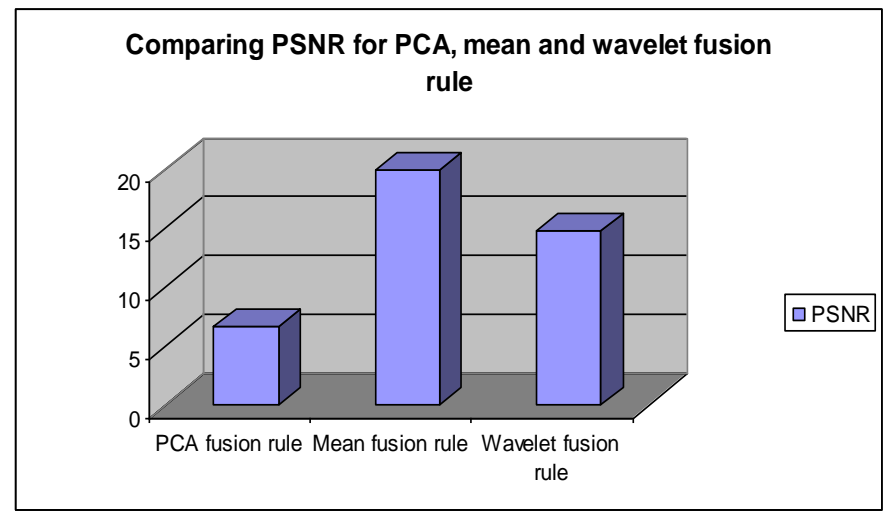

Fig. 8. PSNR for PCA, mean and wavelet fusion rule, mean fusion rules gives high PSNR indicating more contrast and less noise image than PCA and wavelet fusion

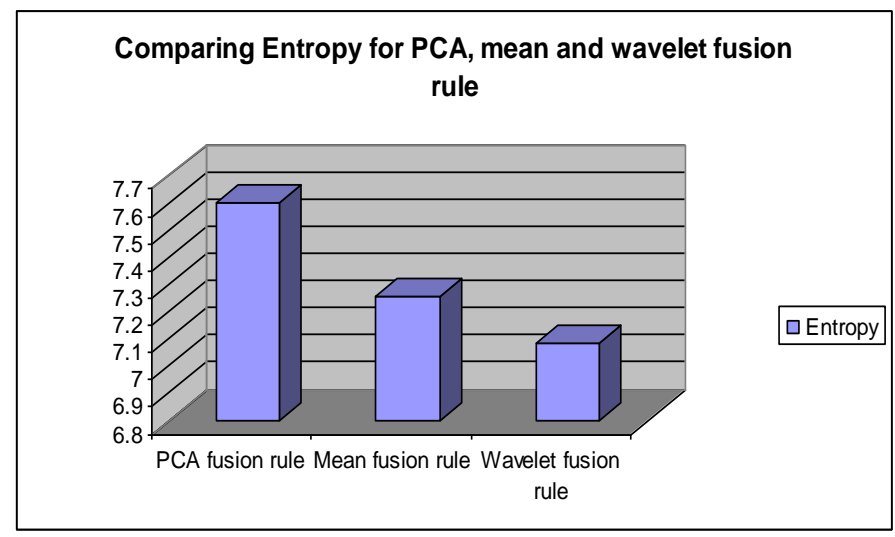

Fig. 9. Entropy for PCA, mean and wavelet fusion rule, PCA is with high entropy than mean and wavelet fusion rule.

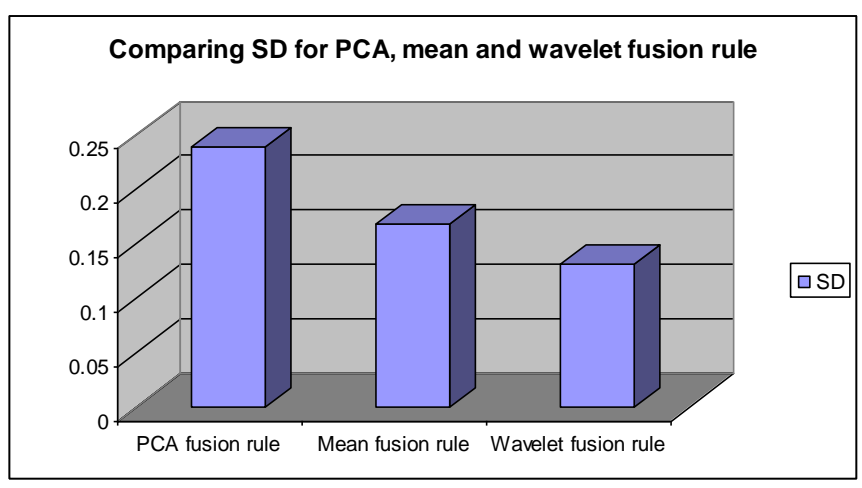

Fig. 10. SD for PCA, mean and wavelet fusion rule PCA is much contrast than mean and wavelet fusion rule
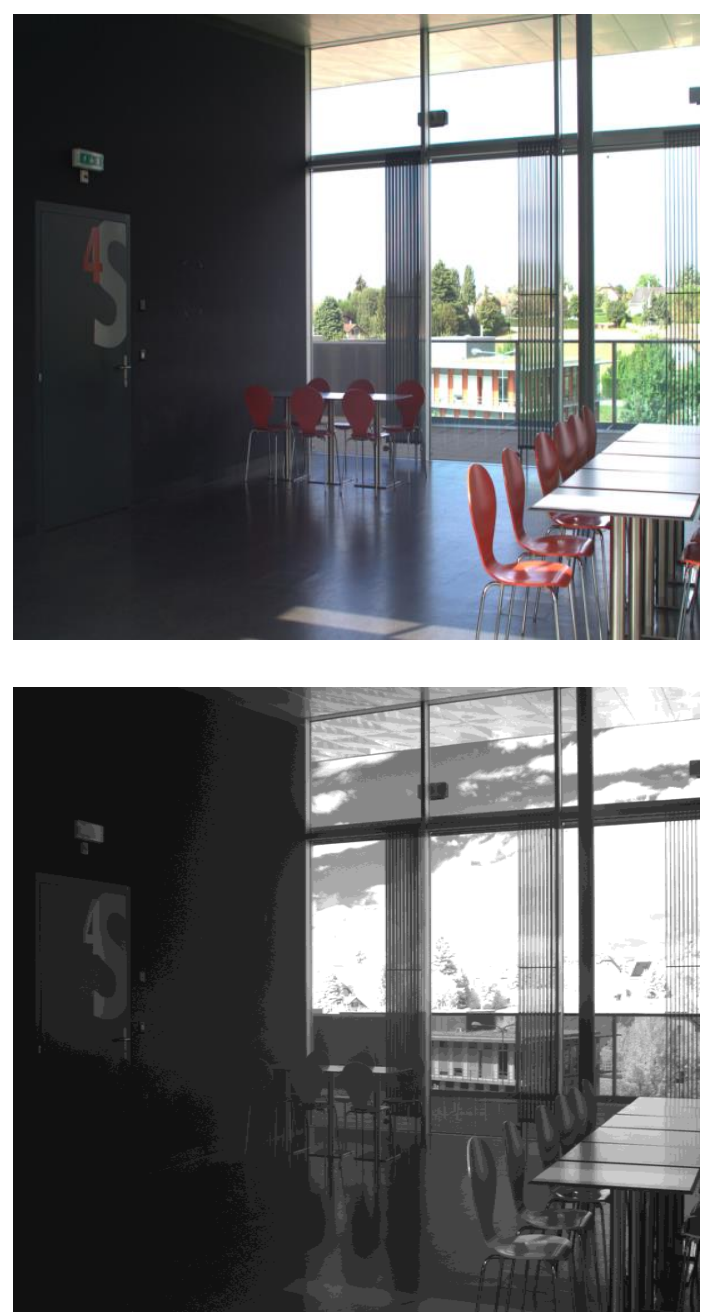

Fig. 11. Two input images for indoor scene one greyscale and the other visual 
Applying these metrics to the second pair of images in Fig. 11 we get those results in table 2

TABLE II. RESULTS OF APPLYING RMSA, PSNR, ENTROPY AND SD TO IMAGES OF FIG. 11

\begin{tabular}{||c|c|c|l|l||}
\hline \hline & RMSA & PSNR & Entropy & SD \\
\hline $\begin{array}{c}\text { PCA fusion } \\
\text { rule }\end{array}$ & $\mathbf{5 9 5 . 3 9 1 0}$ & $\mathbf{2 0 . 3 8 2 8}$ & 7.2164 & 0.3130 \\
\hline $\begin{array}{c}\text { Mean fusion } \\
\text { rule }\end{array}$ & $\mathbf{1 . 6 8 7 8 e + 0 0 3}$ & $\mathbf{1 5 . 8 5 7 7}$ & 7.0609 & 0.3389 \\
\hline $\begin{array}{c}\text { Wavelet fusion } \\
\text { rule }\end{array}$ & $\mathbf{5 3 3 . 4 1 7 0}$ & $\mathbf{2 0 . 8 6 0 1}$ & 7.2073 & 0.3120 \\
\hline
\end{tabular}

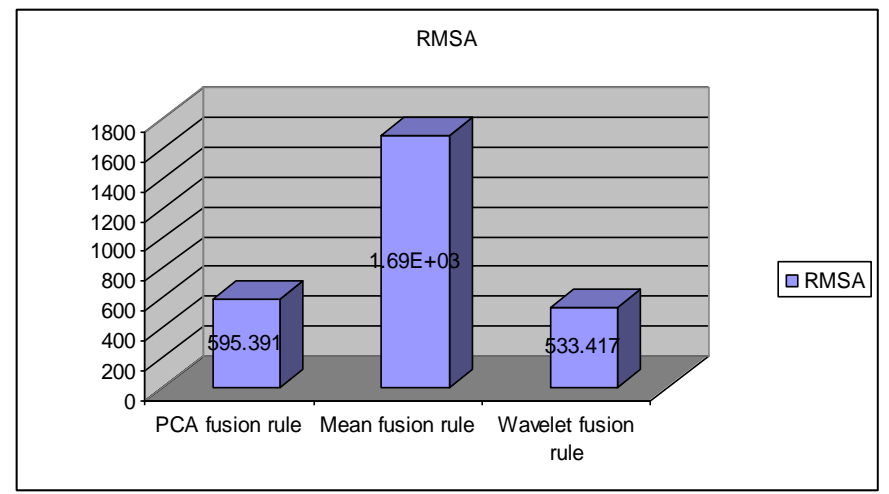

Fig. 12. RMSA for PCA, mean and wavelet fusion rule for table 2, wavelet fusion gives the low RMSA indicating the best fusion quality than the PCA and mean fusion rule

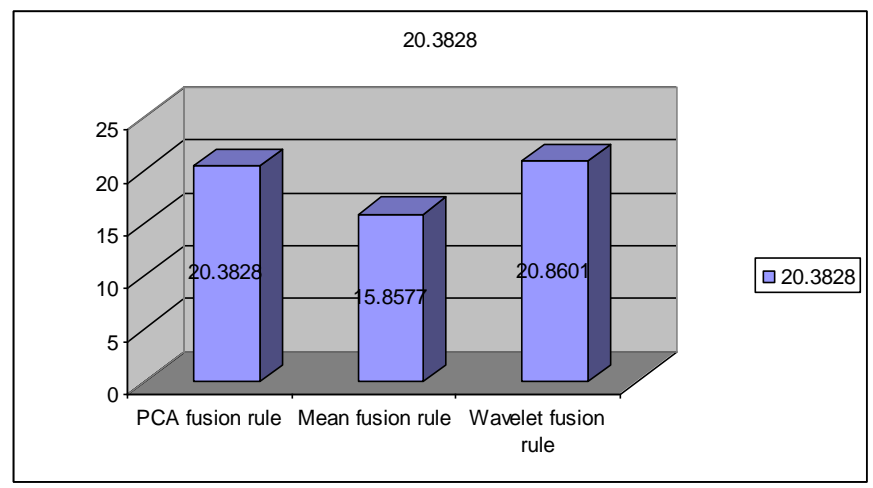

Fig. 13. PSNR for PCA, mean and wavelet fusion rule, wavelet fusion rules gives high PSNR indicating more contrast and less noise image than PCA and wavelet fusion

\section{CONCLUSION}

In this paper we proposed a new image fusion approach for combining a visual colored image and a corresponding grayscale one having some hidden objects or more accurate in some parts of the corresponding visual one; for enhancing the input images we use the curvelet transform approach and used different fusion rules and show a comparison for a three different fusion rule, as for a future work we can use different fields and different type of sensors and apply them to another fusion rules to provide a fused image that provides a detailed description of the details of the scene that appear in one image and not the other and more accurate and high resolution than the input ones.

\section{REFERENCES}

[1] M. Wahed, Ghada EL Taweal, A. Fouad, Gehad M. Taher, Image fusion approach with noise reduction using genetic algorithm, International Journal of Advanced Computer Science and Applications, Vol. 4, No. 11, 2013

[2] Karthik P. Ramesh, Shruti Gupta, and Erik P. Blasch, "Image Fusion Experiment for Information Content", Information Fusion, 10th International Conference on, Vol. 9, 2007.

[3] Y. Zhang, Methods for image fusion quality assessment - a review, comparison and analysis, The International Archives of the Photogrammetry, Remote Sensing and Spatial Information Sciences XXXVII (B7) (2008) 1101-1110.

[4] A. M. Waxman, M. Aguilar, R. A. Baxter, D.A. Fay, D. B. Ireland, J. P. Racamato, W. D. Ross, Opponent-color fusion of multi-sensor imagery: visible, IR and SAR, Proceedings of IRIS Passive Sensors, vol.1, pp. 4361, 1998.

[5] M. Aguilar, D. A. Fay, W. D. Ross, A. M. Waxman, D. B. Ireland, J. P. Racamato, Real-time fusion of low-light CCD and uncooled IR imagery for color night vision, Proceedings of the SPIE, vol.3364, pp. 124-35, 1998.

[6] A. M. Waxman, M. Aguilar, D. A. Fray, D. B. Ireland, J. P. Racamato, Jr., W. D. Ross, J. E. Carrick, A. N. Gove, M. C. Seibert, E. D. Savoye, R. K. Reich, B. E. Burke, W. H. McGonagle, D. M. Craig, Solid-state color night vision: fusion of low-light visible and thermal infrared imagery, Lincoln Laboratory Journal, vol. 11, no. 1, pp. 41-60, 1998.

[7] M. Aguilar, and J. R. New, Fusion of multi-modality volumetric medical imagery, ISIF 2002, pp. 1206-1212.

[8] Manu V T and Philomina Simon, A Novel Statistical Fusion rule for image fusion and its comparison in non susampled contourlet transform domain and wavelet domain: The International journal of multimedia \& its Applications (IJMA) Vol.4, No.2, April 2012.

[9] Y.Kiran Kumar Technical Specialist- Philips HealthCare, Comparison Of Fusion Techniques Applied To Preclinical Images: Fast Discrete Curvelet Transform Using Wrapping Technique \& Wavelet Transform.Journal of Theoretical and Applied Information Technology 